\title{
Semidefinite Programming and Reachable Sets of Dissipative Bilinear Control Systems
}

\author{
Dionisis Stefanatos and Navin Khaneja*
}

\begin{abstract}
In this manuscript, we investigate optimal control problems which arise in connection with manipulation of dissipative quantum dynamics. These problems motivate the study of a class of dissipative bilinear control systems. For these systems it is shown that the optimal solution and the reachable set can be found by solving a semidefinite program. In practice, solutions to these problems generate optimal methods for control of quantum mechanical phenomena in presence of dissipation. In the area of coherent spectroscopy, this translates into the maximum signal to noise ratio that can be obtained in a spectroscopy experiment.
\end{abstract}

\section{Introduction-Statement of the Problem}

Consider the following optimal control problem. Given the dynamical system below

$$
\left[\begin{array}{c}
\dot{x}_{1} \\
\dot{x}_{2} \\
\dot{y}_{1} \\
\dot{y}_{2}
\end{array}\right]=\left[\begin{array}{cccc}
0 & 0 & -v_{1} & 0 \\
0 & 0 & 0 & -v_{2} \\
v_{1} & 0 & -k & -J \\
0 & v_{2} & J & -k
\end{array}\right]\left[\begin{array}{l}
x_{1} \\
x_{2} \\
y_{1} \\
y_{2}
\end{array}\right]
$$

and starting from the initial state $e_{1}=(1,0,0,0)^{T}$, what is the maximum achievable value of $x_{2}$ and what are the optimal controls $v_{1}(t) \in \Re$ and $v_{2}(t) \in \Re$ that achieve this value? Problems like this are associated with optimal manipulation of quantum mechanical phenomena under dissipation.

${ }^{*}$ D. Stefanatos and N. Khaneja are with the Division of Engineering and Applied Sciences, Harvard University, Cambridge, MA 02138 USA (e-mail: stefanat@fas.harvard.edu; navin@eecs.harvard.edu). 
Specifically, the optimization problem stated above comes from Nuclear Magnetic Resonance (NMR) spectroscopy and is related to optimal control of two coupled spins in presence of transverse relaxation [1]. The state variables $x_{i}, y_{i}$ represent averages of various quantum mechanical spin operators. The available controls $v_{1}(t)$ and $v_{2}(t)$ correspond to the components of the magnetic field in the NMR experimental setup. Parameter $k>0$ expresses the transverse relaxation rate while $J$ is the coupling constant between the spins.

Observe that if $v_{1}$ and $v_{2}$ are set to 0 then the initial state $e_{1}$ doesn't evolve at all and there is no build up of $x_{2}$. However, by turning on $v_{1}$, it is possible to rotate $x_{1}$ to $y_{1}$, see Fig. 1. This evolves to $y_{2}$ under the skew symmetric matrix

$$
\left[\begin{array}{cc}
0 & -J \\
J & 0
\end{array}\right]
$$

while both $y_{1}$ and $y_{2}$ dissipate under the term

$$
\left[\begin{array}{cc}
-k & 0 \\
0 & -k
\end{array}\right]
$$

The state $y_{2}$ can then be rotated to $x_{2}$ by switching on the control $v_{2}$. We want to find the optimal $v_{1}$ and $v_{2}$ that maximize the value of $x_{2}$. It is intuitively clear that no matter how large we make $v_{1}(t), v_{2}(t)$, the transfer $x_{1} \rightarrow x_{2}$ cannot be done without any loss, since the intermediate transfer $y_{1} \rightarrow y_{2}$ is entirely due to internal dynamics over which there is no control, thus there is an unavoidable dissipation because of $k>0$.

Define

$$
r_{i}=\sqrt{x_{i}^{2}+y_{i}^{2}} .
$$

Using (11), evolution equations for $r_{1}, r_{2}$ can be found. We get the system

$$
\left[\begin{array}{c}
\dot{r}_{1} \\
\dot{r}_{2}
\end{array}\right]=\left[\begin{array}{cc}
-k \cos ^{2} \phi_{1} & -J \cos \phi_{1} \cos \phi_{2} \\
J \cos \phi_{1} \cos \phi_{2} & -k \cos ^{2} \phi_{2}
\end{array}\right]\left[\begin{array}{c}
r_{1} \\
r_{2}
\end{array}\right] \text {, }
$$

where $\cos \phi_{1}=y_{1} / r_{1}, \cos \phi_{2}=y_{2} / r_{2}$, see Fig. 1] Using the control $v_{1}$ which rotates $x_{1}$ to $y_{1}$, we can control the angle $\phi_{1}$. Analogously, using $v_{2}$ which rotates $y_{2}$ to $x_{2}$, we can control the angle $\phi_{2}$. Denoting $u_{1}=\cos \phi_{1}, u_{2}=$ $\cos \phi_{2}$ and dilating time by a factor of $J$, the above system can be rewritten as

$$
\left[\begin{array}{c}
\dot{r}_{1} \\
\dot{r}_{2}
\end{array}\right]=\left[\begin{array}{cc}
-\xi u_{1}^{2} & -u_{1} u_{2} \\
u_{2} u_{1} & -\xi u_{2}^{2}
\end{array}\right]\left[\begin{array}{l}
r_{1} \\
r_{2}
\end{array}\right]
$$

Here $u_{1}$ and $u_{2}$ are control parameters which take their values in the interval $[-1,1]$ and $\xi=k / J$. The initial problem of maximum transfer from $x_{1}$ to $x_{2}$ has been transformed to the following equivalent question: 


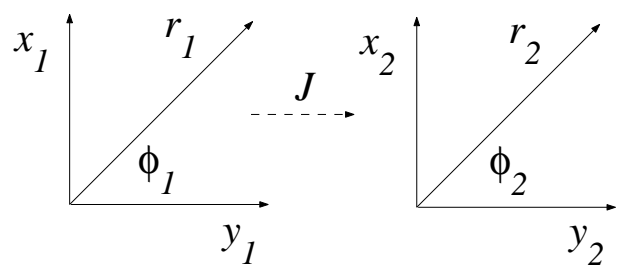

Figure 1: Schematic representation of the evolution of system (11). Control $v_{1}$ rotates $x_{1}$ to $y_{1}$. Under the $J$ coupling $y_{1}$ evolves to $y_{2}$, while both dissipate because of the relaxation term $k$. Control $v_{2}$ rotates $y_{2}$ to $x_{2}$. The new state variables $r_{1}, r_{2}$, defined by (2), are also shown. The corresponding new control parameters are $u_{1}=\cos \phi_{1}, u_{2}=\cos \phi_{2}$.

Given the dynamical system (3) and the initial state $\left(r_{1}(0), r_{2}(0)\right)=$ $(1,0)$, find the optimal control $\left(u_{1}(t), u_{2}(t)\right),\left|u_{1}\right|,\left|u_{2}\right| \leq 1$, such that $r_{2}$ is maximized.

Note that once $r_{2}$ is maximized, the control $v_{2}$ can be used to transfer it to $x_{2}$ with no loss, so the above question is indeed equivalent to the original problem.

Motivated by this example, which originates from a real physical system, let us consider the following $n$-dimensional generalization of system (11):

$$
\left[\begin{array}{l}
\dot{x} \\
\dot{y}
\end{array}\right]=\left[\begin{array}{cc}
0 & -V \\
V & A
\end{array}\right]\left[\begin{array}{l}
x \\
y
\end{array}\right],
$$

where $x=\left(x_{1}, x_{2}, \ldots, x_{n}\right)^{T}, y=\left(y_{1}, y_{2}, \ldots, y_{n}\right)^{T}, V=\operatorname{diag}\left(v_{1}, v_{2}, \ldots, v_{n}\right)$ and $A=\left\{a_{i j}\right\}$ is such that its symmetric part $A+A^{T}$ is negative definite. This condition insures that the norm of the vector $(x, y)$ can only decrease. This models the physics in open quantum systems, where dissipation can only reduce coherence in the system dynamics. Furthermore, $A$ is such that any two states $y_{i}$ and $y_{j}$ are coupled by its off-diagonal elements, not necessarily directly (we say $A$ is irreducible).

Problem 1 Given the dynamical system (4) and the starting state $(x(0), y(0))$, find the optimal control $\left(v_{1}(t), v_{2}(t), \ldots, v_{n}(t)\right)$ which maximizes $x_{n}$.

It is shown in the following section that the negative definiteness condition on $A$ is a sufficient condition for the existence of an optimal solution. 
If we define $r_{i}=\sqrt{x_{i}^{2}+y_{i}^{2}}$ and work as in the 2-dimensional case, we find that $r_{i}$ satisfies the equation

$$
\frac{d r_{i}}{d t}=\sum_{j=1}^{n} a_{i j} u_{i} u_{j} r_{j}
$$

where $u_{i}=y_{i} / r_{i}$. Problem 1 has been transformed to the following.

Problem 2 Given the dynamical system defined by (5) for $i=1,2, \ldots, n$, with $A=\left\{a_{i j}\right\}$ irreducible and such that $A+A^{T}$ negative definite, and the starting state $\left(r_{1}(0), r_{2}(0), \ldots, r_{n}(0)\right)$, with $r_{i}(0) \geq 0$, find the optimal control $\left(u_{1}(t), u_{2}(t), \ldots, u_{n}(t)\right),\left|u_{i}\right| \leq 1$, which maximizes $r_{n}$, while it preserves $r_{i}(t) \geq 0$.

Observe that if $T_{1}<T_{2}$, then the maximum achievable value in time $T_{1}$ cannot exceed the corresponding value in time $T_{2}$, since by putting $u_{i}=0$ the evolution in the interval $\left(T_{1}, T_{2}\right]$ can be stopped. Therefore, Problem 2 is considered as an infinite horizon problem.

Multiplying the $i^{\text {th }}$ equation of system (5) with $2 r_{i}$, we get

$$
\frac{d}{d t}\left(r_{i}^{2}\right)=\sum_{j=1}^{n} 2 a_{i j} u_{i} u_{j} r_{i} r_{j}
$$

and from this

$$
\frac{d}{d t}\left(r_{i}^{2}\right)=U^{2} \sum_{j=1}^{n} 2 a_{i j} \frac{u_{i} r_{i}}{U} \frac{u_{j} r_{j}}{U}
$$

where

$$
U=\sqrt{\sum_{i=1}^{n}\left(u_{i} r_{i}\right)^{2}}
$$

By setting

$$
p_{i}=r_{i}^{2}, \quad m_{i}=\frac{u_{i} r_{i}}{U}
$$

and rescaling time according to $d t^{\prime}=U^{2} d t$, equation (17) becomes

$$
\frac{d p_{i}}{d t^{\prime}}=\sum_{j=1}^{n} 2 a_{i j} m_{i} m_{j}
$$

The initial optimal control problem has been transformed to the following one. 
Problem 3 Given the dynamical system defined by (10) for $i=1,2, \ldots, n$ and the starting point $p(0)=\left(p_{1}(0), p_{2}(0), \ldots, p_{n}(0)\right)^{T}, p_{i}(0) \geq 0$, find the unit vector $m\left(t^{\prime}\right)=\left(m_{1}\left(t^{\prime}\right), m_{2}\left(t^{\prime}\right), \ldots, m_{n}\left(t^{\prime}\right)\right)^{T}$ that maximizes $p_{n}$, while it preserves $p_{i}\left(t^{\prime}\right) \geq 0$. Matrix $A=\left\{a_{i j}\right\}$ is irreducible and such that $A+A^{T}$ is negative definite.

Note that, although Problem 2 is an infinite horizon problem, Problem 3 defined above may achieve its maximum for a finite final time $T_{f}$. There is no inconsistency here, since the times for the two systems are related through $d t^{\prime}=U^{2} d t$, so $T_{f}=\int_{0}^{T_{f}} d t^{\prime}=\int_{0}^{\infty} U^{2} d t$. If $U(t) \rightarrow 0$ sufficiently fast as $t \rightarrow \infty$, then $T_{f}$ is finite. As we will see, this is indeed the case.

In the following, we study problems 2 and 3 in detail. Having found an optimal solution for the latter, we can easily find a corresponding optimal control law for the former. The structure of the paper is as follows. In section 2. it is shown that the solution of Problem 3 can be reduced to the solution of a semidefinite program and that the negative definiteness of the symmetric part of $A$ is a sufficient condition for the existence of an optimal solution. It is also shown how the semidefinite programming formalism can be used for calculating reachable sets. In section 3, some useful existent results regarding the rank of matrices that solve our semidefinite program are presented. These results are used in section 4 , where some specific examples are examined. The examples include system (3) and another system which again arises from an optimal control problem of spin dynamics in NMR spectroscopy.

\section{Reduction to a Semidefinite Program}

In the following, the inner product $\langle\cdot, \cdot\rangle$ in the space of symmetric $n \times n$ matrices $\operatorname{Sym}_{n}$ is defined in the usual way as the trace of the matrix product, i.e. $\langle A, B\rangle=\operatorname{tr}(A B)$ for $A, B \in \operatorname{Sym}_{n}$. Note also that $A \succeq 0$ denotes that matrix $A \in \operatorname{Sym}_{n}$ is positive semidefinite, $A \prec 0$ that is negative definite etc.

Theorem 1 Let us define matrices $A_{i} \in S_{\text {Sym }}, i=1,2, \ldots, n$, by the relation

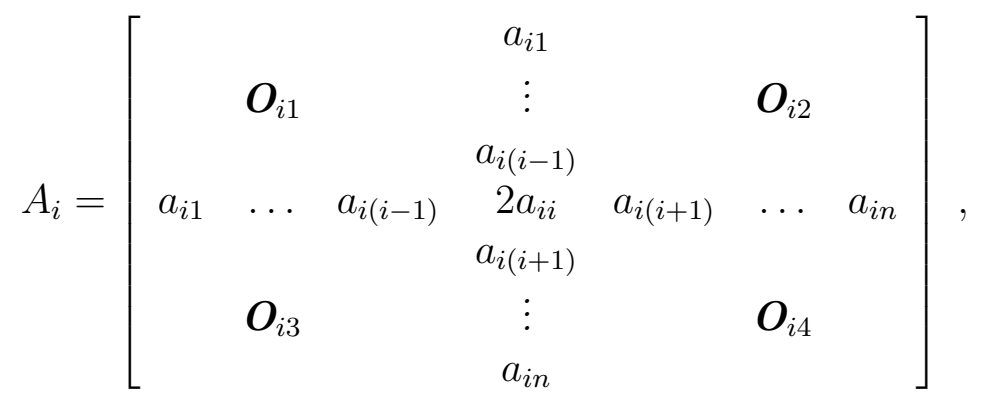


where $a_{i j}$ are the elements of matrix $A$ given in Problem 3 and $\boldsymbol{O}_{i l}, l=$ 1, 2, 3, 4, are zero matrices with appropriate sizes. The solution of Problem 3 can be reduced to the solution of the following semidefinite program:

$$
\begin{gathered}
\text { Find } \mathcal{E}=\max _{M}\left\langle A_{n}, M\right\rangle \\
\text { subject to }\left\langle A_{i}, M\right\rangle=-p_{i}(0), \quad i=1,2, \ldots, n-1 \\
\text { and } \quad M \succeq 0 .
\end{gathered}
$$

The maximum achievable value of $p_{n}$ is $p_{n}(0)+\mathcal{E}$.

Proof: Let $T_{f}$ be the time when $p_{n}$ achieves its maximum, i.e. the final time. From equation (10) it is

$$
p_{i}\left(T_{f}\right)=p_{i}(0)+\sum_{j=1}^{n} 2 a_{i j} \int_{0}^{T_{f}} m_{i}\left(t^{\prime}\right) m_{j}\left(t^{\prime}\right) d t^{\prime} .
$$

Observe that if we define the positive semidefinite matrix $M$ through the relation

$$
M=\int_{0}^{T_{f}} m\left(t^{\prime}\right) m^{T}\left(t^{\prime}\right) d t^{\prime},
$$

then (11) becomes

$$
p_{i}\left(T_{f}\right)=p_{i}(0)+\left\langle A_{i}, M\right\rangle .
$$

One other important observation is that the end point of the optimal trajectory should lie on the line $\left(0,0, \ldots, 0, p_{n}\right)$ in $p$-space. Suppose that the end point has a component $p_{k}>0$ for some $k \neq n$. If $p_{k}$ is directly coupled to $p_{n}$ then choose $m=\left(0,0, \ldots, 0, m_{k}, 0, \ldots, 0, m_{n}\right)^{T}$ such that $m_{n}\left(a_{n k} m_{k}+\right.$ $\left.a_{n n} m_{n}\right)>0$ and evolve the system until $p_{k}=0$. Thereby we get a greater value of $p_{n}$. If $p_{k}$ is not directly coupled to $p_{n}$, we can still transfer from $p_{k}$ to $p_{n}$ using intermediate states (because matrix $A$ is irreducible). We conclude that at the final time $T_{f}$ the end point of the optimal trajectory should lie on the line $\left(0,0, \ldots, 0, p_{n}\right)$. Thus, we have to maximize $p_{n}\left(T_{f}\right)=p_{n}(0)+\left\langle A_{n}, M\right\rangle$ under the conditions $p_{i}\left(T_{f}\right)=p_{i}(0)+\left\langle A_{i}, M\right\rangle=0, i=1,2, \ldots, n-1$. Equivalently, we have to solve the following semidefinite program: Find $\mathcal{E}=$ $\max _{M}\left\langle A_{n}, M\right\rangle$ subject to $\left\langle A_{i}, M\right\rangle=-p_{i}(0)$ for $i=1,2, \ldots, n-1$ and $M \succeq 0$.

Having found an optimal $M$, we can always find an appropriate unit vector $m\left(t^{\prime}\right)$ such that $M=\int_{0}^{T_{f}} m\left(t^{\prime}\right) m^{T}\left(t^{\prime}\right) d t^{\prime}$ and $p_{i}\left(t^{\prime}\right) \geq 0$. Since $M \succeq 0$, it can always be decomposed in the form

$$
M=\sum_{k=1}^{r} \lambda_{k} \mathbf{m}_{k} \mathbf{m}_{k}^{T}
$$


where $\lambda_{k}$ are the positive eigenvalues of $M, \mathbf{m}_{k}$ are the corresponding (real) normalized eigenvectors and $r$ is the rank of $M$. Now let $N$ be a positive integer. Rewrite the above relation in the form

$$
M=N \sum_{k=1}^{r} \Delta \lambda_{k} \mathbf{m}_{k} \mathbf{m}_{k}^{T},
$$

where $\Delta \lambda_{k}=\lambda_{k} / N$, and define the times $t_{k}^{\prime}$ through

$$
t_{0}^{\prime}=0, \quad t_{k}^{\prime}=\sum_{l=1}^{k} \Delta \lambda_{l} \quad \text { for } \quad k=1,2, \ldots, r .
$$

Let us forget for a moment the restrictions $p_{i}\left(t^{\prime}\right) \geq 0$. If we apply the control

$$
m\left(t^{\prime}\right)=\mathbf{m}_{k} \quad \text { for } \quad t_{k-1}^{\prime} \leq t^{\prime}<t_{k}^{\prime}, \quad k=1,2, \ldots, r
$$

and repeat for $N$ times, then on the one hand the requirement

$$
\int_{0}^{T_{f}} m\left(t^{\prime}\right) m^{T}\left(t^{\prime}\right) d t^{\prime}=M
$$

is satisfied and on the other hand the trajectory in $p$-space approximates the line joining the initial point $I\left(p_{1}(0), p_{2}(0), \ldots, p_{n}(0)\right)$ to the final point $F\left(0,0, \ldots, p_{n}\left(T_{f}\right)\right)$, see Fig 2 (a). If $N$ is large enough then the trajectory actually follows this line, see Fig. 2(b), thus the restrictions $p_{i}\left(t^{\prime}\right) \geq 0$ are satisfied. Note that $T_{f}=\sum_{k=1}^{r} \lambda_{k}=\operatorname{tr}(M)$ is finite, if $\operatorname{tr}(M)<+\infty$. In the special case where $r=1$, it is $M=\lambda \mathbf{m m}^{T}$ and thus $m\left(t^{\prime}\right)=\mathbf{m}$ for $t^{\prime} \in\left[0, T_{f}\right], T_{f}=\lambda$.

The conclusion is that we just need to solve the semidefinite program defined above. The maximum achievable value of $p_{n}$ is $p_{n}\left(T_{f}\right)=p_{n}(0)+\mathcal{E}$.

We show next how this control law can be applied to system (5D) in Problem [2. For $0 \leq t^{\prime} \leq t_{1}^{\prime}, m\left(t^{\prime}\right)=\mathbf{m}_{1}=$ constant. Since, additionally, $\mathbf{m}_{1}$ is a unit vector, we can assume without loss of generality that its first component $m_{1} \neq 0$. Consider the ratios

$$
\frac{u_{i} r_{i}}{u_{1} r_{1}}=\frac{m_{i}\left(t^{\prime}\right)}{m_{1}\left(t^{\prime}\right)}=s_{i}, \quad i=1,2, \ldots, n
$$

For $0 \leq t^{\prime} \leq t_{1}^{\prime}, s_{i}$ are constant. Define

$$
\mathcal{M}=\max _{i}\left(\left|\frac{s_{i} r_{1}}{r_{i}}\right|\right), \quad i=1,2, \ldots, n .
$$




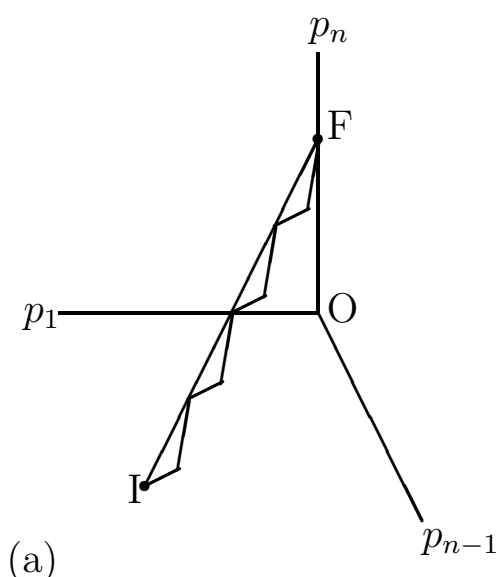

(a)

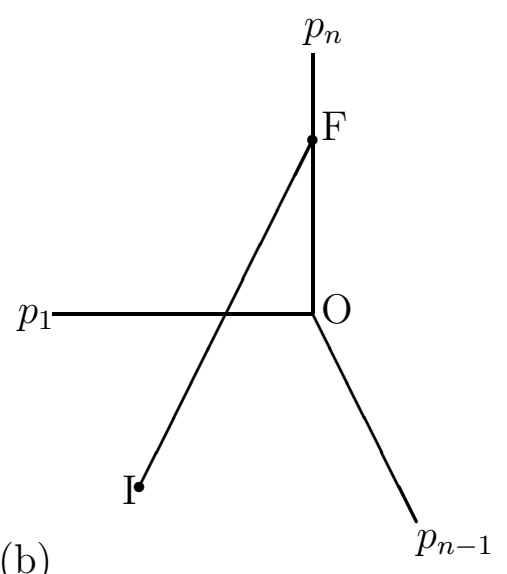

(b)

Figure 2: (a) Trajectory in $p$-space following the control law presented in the text, for $r=2$ and $N=4$. It approximates the straight line from the initial point $I$ to the final point $F$. Note that the restrictions $p_{i}\left(t^{\prime}\right) \geq 0$ may not be satisfied when $N$ is small (b) For large $N$ the trajectory coincides with the line $I F$, so the restrictions $p_{i}\left(t^{\prime}\right) \geq 0$ are satisfied.

The optimal policy can be realized as

$$
u_{1}=\frac{1}{\mathcal{M}}
$$

and

$$
u_{i}=\frac{s_{i} r_{1}}{r_{i}} u_{1}
$$

where $i=2,3, \ldots, n$. With the above choice we insure that $\left|u_{i}\right| \leq 1$. Using this feedback law we can evolve system (15) in time $t$ and calculate the function $U(t)=\sum_{i=1}^{n}\left(u_{i} r_{i}\right)^{2}$. Then, we can find $t^{\prime}=\int_{0}^{t} U^{2} d t$. When $t^{\prime}=t_{1}^{\prime}$, we switch to $m\left(t^{\prime}\right)=\mathbf{m}_{2}$ and repeat the above procedure. If the rank of $M$ is $r=1$ then the ratios $s_{i}$ keep the same value for all times. Note that the maximum achievable value of $r_{n}$ is

$$
r_{n}(\infty)=\sqrt{p_{n}\left(T_{f}\right)}=\sqrt{p_{n}(0)+\mathcal{E}}=\sqrt{r_{n}^{2}(0)+\mathcal{E}} .
$$

In the above discussion we implicitly assumed that an optimal solution exists, and we used for $\mathcal{E}$ the characterization "maximum" instead of the more formal "supremum". We show below that the negative definiteness of $A+A^{T}$ is a sufficient condition for the existence of an optimal solution. The following lemma is used.

Lemma 1 If $B \succ 0$ and $M \succeq 0, B, M \in \operatorname{Sym}_{n}$, then $\langle B, M\rangle \geq 0$. 
Proof: Since $B \in \mathrm{Sym}_{n}$ it can be diagonalized by an orthogonal matrix $O, B=O \Delta O^{T}$, where $\Delta=\operatorname{diag}\left(\lambda_{1}, \lambda_{2}, \ldots, \lambda_{n}\right)$ and $\lambda_{i}>0$ are the eigenvalues of the positive definite matrix $B$. It is

$$
\langle B, M\rangle=\operatorname{tr}(B M)=\operatorname{tr}\left(O \Delta O^{T} M\right)=\operatorname{tr}\left(\Delta O^{T} M O\right)=\operatorname{tr}(\Delta \tilde{M})=\sum_{i=1}^{n} \lambda_{i} \tilde{m}_{i i},
$$

where $\tilde{M}=O^{T} M O$ and $\tilde{m}_{i i}$ are its diagonal elements. But $\tilde{M}^{T}=\tilde{M}$ and $x^{T} \tilde{M} x=(O x)^{T} M O x \geq 0$ for every $x \in \Re^{n}$, since $M \succeq 0$. So, it is also $\tilde{M} \succeq 0$ and thus $\tilde{m}_{i i} \geq 0$. Since, additionally, $\lambda_{i}>0$, we conclude that $\langle B, M\rangle=\sum_{i=1}^{n} \lambda_{i} \tilde{m}_{i i} \geq 0$.

Theorem 2 If $A+A^{T} \prec 0$ then the semidefinite program defined in Theorem 1 has an optimal solution.

Proof: First we show that the set $S$ of all matrices $M \succeq 0$ satisfying the equality constraints $\left\langle A_{i}, M\right\rangle=-p_{i}(0), i=1,2, \ldots, n-1$, is non-empty. Indeed, the matrix

$$
M=\operatorname{diag}\left(-p_{1}(0) / 2 a_{11},-p_{2}(0) / 2 a_{22}, \ldots,-p_{n}(0) / 2 a_{n n}\right)
$$

satisfies these conditions and, additionally, it is $M \succeq 0$, since $p_{i}(0) \geq 0$ and $a_{i i}<0\left(A+A^{T} \prec 0\right)$. Note that $S$ is closed and convex. Now consider the function $f: S \rightarrow \Re$ defined by $f(M)=\left\langle A_{n}, M\right\rangle$ and the matrix $B=$ $-\sum_{i=1}^{n} A_{i}=-\left(A+A^{T}\right) \succ 0$. From Lemma 1 and for $M \in S$, we have

$$
\langle B, M\rangle \geq 0 \Rightarrow\left\langle A_{n}, M\right\rangle \leq-\sum_{i=1}^{n-1}\left\langle A_{i}, M\right\rangle=\sum_{i=1}^{n-1} p_{i}(0)<+\infty \Rightarrow f(M)<+\infty .
$$

Thus $\sup _{M \in S} f(M)<+\infty$ and since $S$ is closed the supremum is achieved for a $M_{0} \in S$, so it is actually a maximum. The existence of an optimal solution is established.

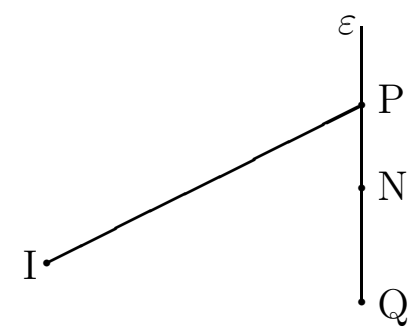

Figure 3: Construction of the reachable set of point I. 
We finally show how the semidefinite programming formalism can be used for calculating the reachable set of point $I\left(p_{1}(0), p_{2}(0), \ldots, p_{n}(0)\right)$. Consider the line $\varepsilon$ parallel to $p_{n}$-axis, with $p_{i}=$ constant $\geq 0, i=1,2, \ldots, n-1$. The maximum achievable value of $p_{n}$ on $\varepsilon$, starting from $I$, can be found by solving the following semidefinite program: Find $\max _{M}\left\langle A_{n}, M\right\rangle$ subject to $\left\langle A_{i}, M\right\rangle=p_{i}-p_{i}(0)$ for $i=1,2, \ldots, n-1$ and $M \succeq 0$. If this program has a solution $M_{0}$ such that $p_{n}=p_{n}(0)+\left\langle A_{n}, M_{0}\right\rangle \geq 0$, then let $\mathrm{P}$ be the point $\left(p_{1}, p_{2}, \ldots, p_{n}\right)$ of $\varepsilon$, see Fig. 3. This point belongs to the reachable set of I. Additionally, every point $N\left(p_{1}, p_{2}, \ldots, p_{n-1}, p_{n}^{\prime}\right)$ of $\varepsilon$ with $0 \leq p_{n}^{\prime} \leq p_{n}$, see Fig. 3, belongs also to the reachable set (first arrive at $P$ and then use $m=(0,0, \ldots, 1)^{T}$ to go down, since (10) gives $\dot{p}_{n}=a_{n n}<0, \dot{p}_{i}=0$ for $i \neq n)$. Thus, the segment $P Q$, where $Q\left(p_{1}, p_{2}, \ldots, p_{n-1}, 0\right)$, is in the reachable set. By repeating the above procedure for all the allowed $\varepsilon \| p_{n}$, the reachable set of $I$ can be constructed.

\section{Remarks on the Rank of the Semidefinite Program Solutions}

In the preceding section we saw that the bigger the rank of the optimal $M$ for the semidefinite program, the more complicated is the optimal control law. Thus, it would be useful to know if there exist low rank optimal solutions and, additionally, rank upper bounds for them. Even more, we would like to know for what matrices $A$ the corresponding semidefinite program has solutions of the lowest possible rank $r=1$. In this section we present a series of results in these directions.

Lemma 2 Let us fix $A_{1}, A_{2}, \ldots, A_{k} \in S_{\text {Sym }}$ and $\alpha_{1}, \alpha_{2}, \ldots, \alpha_{k} \in \Re$. If there is a matrix $M \succeq 0$ such that

$$
\left\langle A_{i}, M\right\rangle=\alpha_{i}, \quad i=1,2, \ldots, k,
$$

then there is a matrix $M_{0} \succeq 0$ such that

$$
\left\langle A_{i}, M_{0}\right\rangle=\alpha_{i}, \quad i=1,2, \ldots, k
$$

and, additionally,

$$
\operatorname{rank} M_{0} \leq\left\lfloor\frac{\sqrt{8 k+1}-1}{2}\right\rfloor,
$$

where $\lfloor\cdot\rfloor$ denotes the integer part of the embraced number.

Proof: See 2], chapter II, proposition 13.1. 
Proposition 1 If $A+A^{T} \prec 0$ then there is an optimal solution $M_{0}$ to the semidefinite program defined in Theorem $\mathbf{1}$, with

$$
\operatorname{rank} M_{0} \leq\left\lfloor\frac{\sqrt{8 n+1}-1}{2}\right\rfloor .
$$

Proof: From Theorem 2 we have that, since $A+A^{T} \prec 0$, the semidefinite program has an optimal solution $M \succeq 0$, which satisfies

$$
\left\langle A_{i}, M\right\rangle=-p_{i}(0), \quad i=1,2, \ldots, n-1, \quad\left\langle A_{n}, M\right\rangle=\mathcal{E}
$$

According to Lemma 2, there exists a $M_{0} \succeq 0$ such that

$$
\left\langle A_{i}, M_{0}\right\rangle=-p_{i}(0), \quad i=1,2, \ldots, n-1, \quad\left\langle A_{n}, M_{0}\right\rangle=\mathcal{E}
$$

and

$$
\operatorname{rank} M_{0} \leq\left\lfloor\frac{\sqrt{8 n+1}-1}{2}\right\rfloor .
$$

Obviously $M_{0}$ is also an optimal solution.

Corollary 1 If $A+A^{T} \prec 0$ and $A$ is $2 \times 2$, then the semidefinite program has an optimal solution of rank $r \leq 1$.

Proof: Apply Proposition 1 for $n=2$.

In general, the bound imposed by Lemma 2 is the best possible. However, there is one special case where it can be sharpened.

Lemma 3 For some positive integer $r$, let us fix $k=(r+2)(r+1) / 2$ matrices $A_{1}, A_{2}, \ldots, A_{k} \in$ Sym $_{n}$, where $n \geq r+2$, and $k$ numbers $\alpha_{1}, \alpha_{2}, \ldots, \alpha_{k} \in \Re$. If there is a matrix $M \succeq 0$ such that

$$
\left\langle A_{i}, M\right\rangle=\alpha_{i}, \quad i=1,2, \ldots, k
$$

and the set of all such matrices is bounded, then there is a matrix $M_{0} \succeq 0$ such that

$$
\left\langle A_{i}, M_{0}\right\rangle=\alpha_{i}, \quad i=1,2, \ldots, k
$$

and, additionally,

$$
\operatorname{rank} M_{0} \leq r .
$$

Proof: See [2], chapter II, proposition 13.4.

Proposition 2 If $A+A^{T} \prec 0$ and $A$ is $3 \times 3$, then the semidefinite program has an optimal solution of rank $r \leq 1$. 
Proof: Since $A+A^{T} \prec 0$, the semidefinite program has an optimal solution $M \succeq 0$, which satisfies

$$
\left\langle A_{i}, M\right\rangle=-p_{i}(0), \quad i=1,2, \quad\left\langle A_{n}, M\right\rangle=\mathcal{E} .
$$

From Lemma 3 we see that the choice $r=1$ gives $k=3=n$, since $n=3$ according to the hypothesis of the proposition. In order to apply Lemma 3 , we just need to show that the set of optimal matrices, i.e. all the matrices $M \succeq 0$ satisfying the above relations, is bounded. Consider the matrix $B=-\sum_{i=1}^{3} A_{i}=-\left(A+A^{T}\right) \succ 0$. For a matrix $M \succeq 0$ in the set of optimal solutions, we have

$$
\langle B, M\rangle=p_{1}(0)+p_{2}(0)-\mathcal{E}<+\infty .
$$

But from Lemma 1, we have also $\langle B, M\rangle=\sum_{i=1}^{3} \lambda_{i} \tilde{m}_{i i}$, where $\lambda_{i}$ are the eigenvalues of $B$ and $\tilde{m}_{i i}$ the diagonal elements of the matrix $\tilde{M}=O^{T} M O$, $O$ the orthogonal matrix diagonalizing $B$. Combining these we find that

$$
\sum_{i=1}^{3} \lambda_{i} \tilde{m}_{i i}<+\infty .
$$

Since $\lambda_{i}>0$ and $\tilde{m}_{i i} \geq 0$, the above relation implies that

$$
\tilde{m}_{i i}<+\infty
$$

thus

$$
\operatorname{tr}(M)=\operatorname{tr}(\tilde{M})<+\infty
$$

But

$$
\langle M, M\rangle=\operatorname{tr}\left(M^{2}\right) \leq(\operatorname{tr}(M))^{2}<+\infty,
$$

since $M \succeq 0$. So indeed the set of optimal $M$ is bounded and we can apply Lemma 3] with $r=1$. This means that there is an optimal $M_{0} \succeq 0$ with $\operatorname{rank} M_{0} \leq 1$. Note that the bound that Proposition 1 gives in this case is only $\lfloor(\sqrt{8 \cdot 3+1}-1) / 2\rfloor=2$.

Lemma 4 Let us call an $n \times n$ matrix $A=\left\{a_{i j}\right\} r$-diagonal if $a_{i j}=0$ unless $|i-j|<r$. Suppose that the matrices $A_{1}, A_{2}, \ldots, A_{k} \in S y m_{n}$ are $r$-diagonal and there exists a matrix $M \succeq 0$ such that

$$
\left\langle A_{i}, M\right\rangle=\alpha_{i} \in \Re, \quad i=1,2, \ldots, k .
$$

Then there exists a matrix $M_{0} \succeq 0$ such that

$$
\left\langle A_{i}, M_{0}\right\rangle=\alpha_{i}, \quad i=1,2, \ldots, k
$$

and, additionally,

$$
\operatorname{rank} M_{0} \leq r
$$


Proof: See [2], chapter $I V$, corollary 10.3, problem 3.

Proposition 3 If $A+A^{T} \prec 0$ and $A$ is $r$-diagonal, then the semidefinite program has an optimal solution of rank $\leq r$.

Proof: Since $A+A^{T} \prec 0$, there exists an optimal solution of the semidefinite program. Since $A$ is $r$-diagonal, the corresponding $A_{i}$ are also $r$-diagonal. Thus, we can apply Lemma 4, which assures the existence of an optimal solution of rank $\leq r$.

We conclude this section by noting that there is strong numerical evidence that the following conjecture is true.

Conjecture 1 If $A+A^{T} \prec 0$ then the semidefinite program has an optimal solution of rank $r=1$.

\section{Examples}

In this section we solve problems 2 and 3 for some specific systems. We start from the system with

$$
A=\left[\begin{array}{cc}
-\xi & -1 \\
1 & -\xi
\end{array}\right], \xi>0,
$$

which corresponds to system (3) appeared in the introduction. It is not necessary to solve numerically the corresponding semidefinite program, because we can attack this particular case analytically. Since $A+A^{T}=\operatorname{diag}(-2 \xi,-2 \xi) \prec$ 0 and $A$ is $2 \times 2$, from Corollary 1 we have that there is an optimal solution to the semidefinite program with rank $r \leq 1$. Thus, there is an optimal constant vector $m=\left(m_{1}, m_{2}\right)^{T}$, solution to Problem 3. The system equation (10) with $A$ given above and $m$ constant gives

$$
p_{1}\left(T_{f}\right)=p_{1}(0)-\left(\xi m_{1}^{2}+m_{1} m_{2}\right) T_{f}, \quad p_{2}\left(T_{f}\right)=p_{2}(0)+\left(m_{2} m_{1}-\xi m_{2}^{2}\right) T_{f} .
$$

Optimality requires

$$
p_{1}\left(T_{f}\right)=0 \Rightarrow T_{f}=\frac{p_{1}(0)}{\xi m_{1}^{2}+m_{1} m_{2}},
$$

So

$$
p_{2}\left(T_{f}\right)=p_{2}(0)+\frac{m_{2} m_{1}-\xi m_{2}^{2}}{\xi m_{1}^{2}+m_{1} m_{2}} p_{1}(0)
$$


In order to maximize $p_{2}\left(T_{f}\right)$, we just need to maximize the coefficient of $p_{1}(0)$. If we set $m_{2} / m_{1}=x$, then this coefficient takes the form

$$
f(x)=\frac{x-\xi x^{2}}{x+\xi}
$$

Before maximizing $f$, we find the allowed values of variable $x$. It should be $p_{2}\left(T_{f}\right) \geq p_{2}(0) \Rightarrow x-\xi x^{2} \geq 0$ and $p_{1}\left(T_{f}\right) \leq p_{1}(0) \Rightarrow x+\xi \geq 0$. These are both satisfied when $x \in[0,1 / \xi]$. We calculate the maximum of $f$ in this interval. It is not difficult to verify that

$$
f^{\prime}(x)=-\frac{\xi\left(x^{2}+2 \xi x-1\right)}{(x+\xi)^{2}}
$$

becomes zero at the point

$$
x_{0}=\sqrt{1+\xi^{2}}-\xi
$$

of the interval $[0,1 / \xi]$. Also verify that $f^{\prime}(x)>0$ for $x \in\left[0, x_{0}\right)$ and $f^{\prime}(x)<0$ for $x \in\left(x_{0}, 1 / \xi\right]$. So $f\left(x_{0}\right)$ is a maximum in the interval $[0,1 / \xi]$. After some manipulation we find that

$$
f\left(x_{0}\right)=x_{0}^{2} .
$$

The maximum achievable value of $p_{2}$ is

$$
p_{2}\left(T_{f}\right)=p_{2}(0)+x_{0}^{2} p_{1}(0)
$$

and the optimal unit vector is

$$
m=\left(\frac{1}{\sqrt{1+x_{0}^{2}}}, \frac{x_{0}}{\sqrt{1+x_{0}^{2}}}\right) .
$$

The optimal trajectory in $p$-space is a straight line joining the points $\left(p_{1}(0), p_{2}(0)\right)$ and $\left(0, p_{2}\left(T_{f}\right)\right)$.

The maximum achievable value of $r_{2}$ is

$$
r_{2}(\infty)=\sqrt{r_{2}^{2}(0)+x_{0}^{2} r_{1}^{2}(0)} .
$$

If the starting state is the point $\left(r_{1}(0), r_{2}(0)\right)=(1,0)$, the maximum transfer efficiency takes the value

$$
r_{2}(\infty)=x_{0}=\sqrt{1+\xi^{2}}-\xi
$$


For $\xi=1$ this efficiency is $\sqrt{2}-1$. The optimal controls $u_{1}, u_{2}$ for system (3), can be found by using the method described in section 2 If we define

$$
\mathcal{M}=\max \left(1, \frac{x_{0} r_{1}}{r_{2}}\right)
$$

the optimal policy can be realized as

$$
u_{1}=\frac{1}{\mathcal{M}}, \quad u_{2}=\frac{x_{0} r_{1}}{r_{2}} u_{1} .
$$

Observe that the initial point $(1,0)$ is a stationary point of the optimal control policy $\left[r_{2}(0)=0 \Rightarrow \mathcal{M}=\infty \Rightarrow u_{1}=0 \Rightarrow u_{2}=0\right]$. This optimal policy in the infinite horizon case should then be interpreted as the limit of optimal control policy for the corresponding finite time problem [Finite time for the $r_{i}$ system, don't confuse it with the finite time problem for $p_{i}$ which corresponds to the infinite horizon problem for $r_{i}$. It is the special case $k_{1}=k_{2}=0$ of the finite time problem solved in the preceding chapter. The solution for this particular case can be found in [1]. In practice, we give a small but finite value in $r_{2}(0)$ (an initial 'kick' from zero) which makes the optimal control law applicable. In Fig. 4(a) we plot the optimal controls $u_{1}$ and $u_{2}$. In Fig. 4(b) we depict $r_{1}(t), r_{2}(t)$ and in Fig. 世(c) the corresponding optimal trajectory in $r$-space. For all these figures it is $\xi=1$ and $\left(r_{1}(0), r_{2}(0)\right)=(1,0)$.

Remark 1 The closure of the reachable set of point $(1,0)$ is

$$
\overline{\boldsymbol{R}((1,0))}=\left\{r_{1}, r_{2} \geq 0 \mid \sqrt{r_{2}^{2}+x_{0}^{2} r_{1}^{2}} \leq x_{0}\right\}
$$

where $x_{0}=\sqrt{1+\xi^{2}}-\xi$. This set is depicted in Fig. 4 (c) for $\xi=1$. The closure of the reachable set $\overline{\boldsymbol{R}((1,0,0,0))}$ for the corresponding bilinear system (1) is

$$
\left\{\left(x_{1}, x_{2}, y_{1}, y_{2}\right) \in \Re^{4} \mid \sqrt{\left(x_{2}^{2}+y_{2}^{2}\right)+x_{0}^{2}\left(x_{1}^{2}+y_{1}^{2}\right)} \leq x_{0}\right\} .
$$

The next case that we examine is the system with

$$
A=\left[\begin{array}{ccc}
-\xi & -1 & 0 \\
1 & -\xi & -1 \\
0 & 1 & -\xi
\end{array}\right], \xi>0
$$

Since $A+A^{T}=\operatorname{diag}(-2 \xi,-2 \xi,-2 \xi) \prec 0$ and $A$ is $3 \times 3$, from Proposition 2 we have that the semidefinite program has a solution of rank $r \leq 1$. Now 

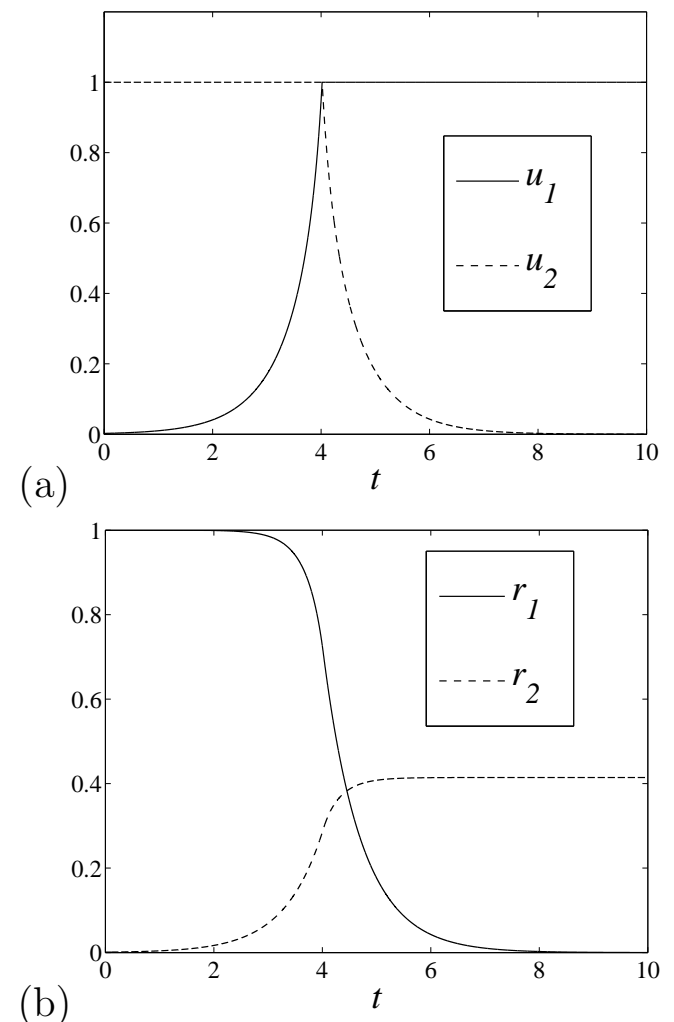

(b)

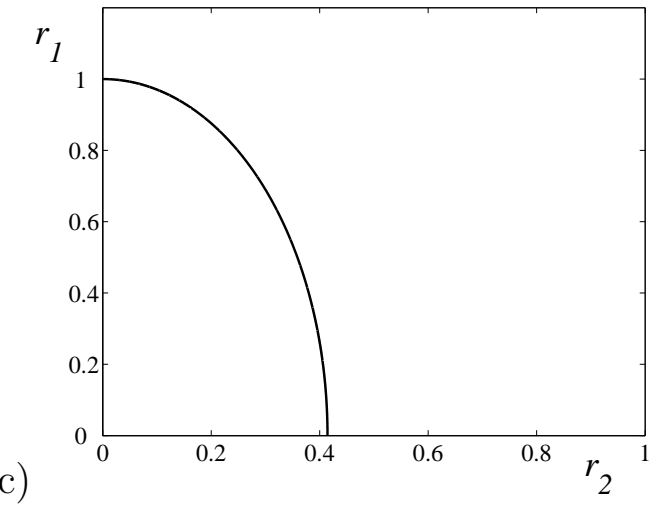

Figure 4: (a) Optimal controls $u_{1}(t)$ and $u_{2}(t)$ for system (3) when $\xi=1$ and $\left(r_{1}(0), r_{2}(0)\right)=(1,0)$ (b) The corresponding state variables $r_{1}(t)$ and $r_{2}(t)$ (c) The optimal trajectory in $r$-space. 
let us become more specific, so set $\xi=1$ and consider the starting point $\left(p_{1}(0), p_{2}(0), p_{3}(0)\right)=(1,1,0)$. The corresponding matrices $A_{i}$ are

$$
A_{1}=\left[\begin{array}{ccc}
-2 & -1 & 0 \\
-1 & 0 & 0 \\
0 & 0 & 0
\end{array}\right], \quad A_{2}=\left[\begin{array}{ccc}
0 & 1 & 0 \\
1 & -2 & -1 \\
0 & -1 & 0
\end{array}\right], \quad A_{3}=\left[\begin{array}{ccc}
0 & 0 & 0 \\
0 & 0 & 1 \\
0 & 1 & -2
\end{array}\right]
$$

If we solve numerically the corresponding semidefinite program using some appropriate software package, for example SDPT3 [3], we find that the optimal matrix $M \succeq 0$ is

$$
M=\left[\begin{array}{lll}
0.1775 & 0.3225 & 0.1304 \\
0.3225 & 0.5856 & 0.2368 \\
0.1304 & 0.2368 & 0.0958
\end{array}\right]
$$

and the maximum achievable value of $p_{3}$ is

$$
p_{3}\left(T_{f}\right)=p_{3}(0)+\left\langle A_{3}, M\right\rangle=\left\langle A_{3}, M\right\rangle=0.2821 \text {. }
$$

It is easy to verify that this matrix has two zero eigenvalues and one nonzero, so its rank is indeed $r=1$. It can be written in the form $M=\lambda m m^{T}$, where $\lambda=0.8589\left(=T_{f}\right)$ is the nonzero eigenvalue and

$$
m=\left(m_{1}, m_{2}, m_{3}\right)^{T}=(0.4546,0.8257,0.3339)^{T}
$$

the corresponding eigenvector. This unit vector is the optimal solution for Problem 3 .

The maximum achievable value of $r_{3}$ is $r_{3}(\infty)=\sqrt{p_{3}\left(T_{f}\right)}=0.5311$. We find the optimal $u_{1}, u_{2}, u_{3}$. Let us set

$$
x_{0}=\frac{m_{2}}{m_{1}}=1.8163, \quad y_{0}=\frac{m_{3}}{m_{1}}=0.7345 \text {. }
$$

If we define

$$
\mathcal{M}=\max \left(1, \frac{x_{0} r_{1}}{r_{2}}, \frac{y_{0} r_{1}}{r_{3}}\right)
$$

then the optimal policy can be realized as

$$
u_{1}=\frac{1}{\mathcal{M}}, \quad u_{2}=\frac{x_{0} r_{1}}{r_{2}} u_{1}, \quad u_{3}=\frac{y_{0} r_{1}}{r_{3}} u_{1} .
$$

Observe that the initial point $(1,1,0)$ is a stationary point of the optimal control policy $\left[r_{3}(0)=0 \Rightarrow \mathcal{M}=\infty \Rightarrow u_{1}=0 \Rightarrow u_{2}=u_{3}=0\right]$. The situation is similar with that in the previous example. Again, the optimal policy in the infinite horizon case should be interpreted as the limit of the 


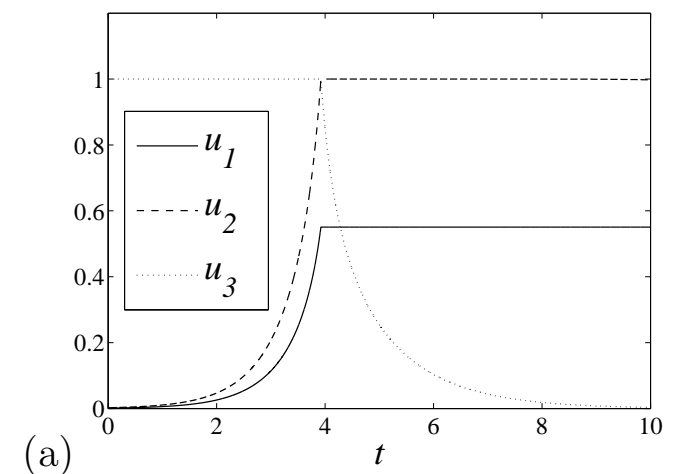

(a)

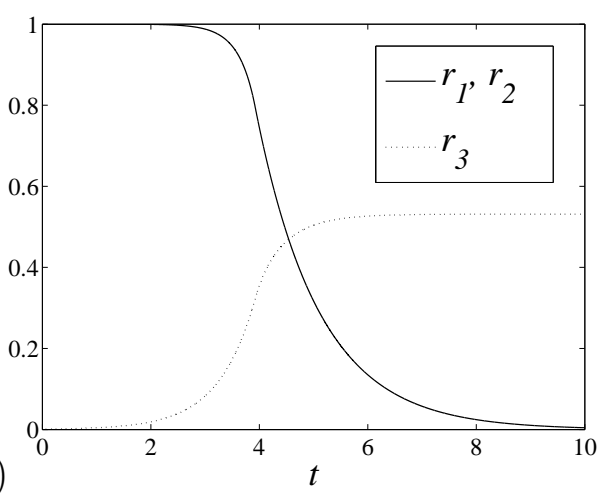

(c)

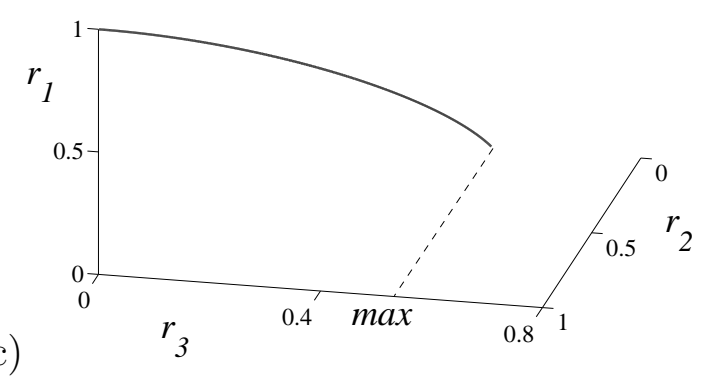

Figure 5: (a) Optimal controls $u_{1}(t), u_{2}(t)$ and $u_{3}(t)$ for system (15), with $A$ the $3 \times 3$ matrix given in the text, when $\xi=1$ and $\left(r_{1}(0), r_{2}(0), r_{3}(0)\right)=$ $(1,1,0)$ (b) The corresponding state variables $r_{1}(t), r_{2}(t)$ and $r_{3}(t)$. Observe that $r_{2}(t) / r_{1}(t)=1$ throughout. Remember that the optimal trajectory in $p$-space is a straight line ending at the point $\left(0,0, p_{3}\left(T_{f}\right)\right)$, so $p_{2}(t) / p_{1}(t)=$ $p_{2}(0) / p_{1}(0)=1$ for the starting point $(1,1,0)$ (c) The optimal trajectory in $r$-space. 
optimal policy for the corresponding finite time problem. In practice, a small but finite value is given to $r_{3}(0)$. In Fig. 5(a) we plot the optimal controls $u_{1}, u_{2}$ and $u_{3}$. In Fig. 5(b) we depict $r_{1}(t), r_{2}(t), r_{3}(t)$ and in Fig. 5)(c) the corresponding optimal trajectory in $r$-space.

Another interesting case to examine is the same system with $\xi>0$ unspecified and starting point $\left(p_{1}(0), 0, p_{3}(0)\right)$. This problem can be solved analytically and has the practical application that it gives an upper bound for our ability to coherently control a specific dissipative quantum system 4 . As before, we know that there is an optimal constant vector $m=\left(m_{1}, m_{2}, m_{3}\right)^{T}$. From equations (10) we find

$$
\begin{aligned}
& p_{1}\left(T_{f}\right)=p_{1}(0)-\left(\xi m_{1}^{2}+m_{1} m_{2}\right) T_{f}, \\
& p_{2}\left(T_{f}\right)=p_{2}(0)+\left(m_{2} m_{1}-\xi m_{2}^{2}-m_{2} m_{3}\right) T_{f}, \\
& p_{3}\left(T_{f}\right)=p_{3}(0)+\left(m_{3} m_{2}-\xi m_{3}^{2}\right) T_{f} .
\end{aligned}
$$

Optimality requires

$$
p_{1}\left(T_{f}\right)=0 \Rightarrow T_{f}=\frac{p_{1}(0)}{\xi m_{1}^{2}+m_{1} m_{2}}
$$

and

$$
p_{2}\left(T_{f}\right)=0 \Rightarrow m_{2} m_{1}-\xi m_{2}^{2}-m_{2} m_{3}=0 .
$$

So, we have to maximize

$$
p_{3}\left(T_{f}\right)=p_{3}(0)+\frac{m_{3} m_{2}-\xi m_{3}^{2}}{\xi m_{1}^{2}+m_{1} m_{2}} p_{1}(0)
$$

subject to the constraint (28). We just need to maximize the coefficient of $p_{1}(0)$ under the same condition. If we set

$$
\frac{m_{2}}{m_{1}}=x, \quad \frac{m_{3}}{m_{1}}=y,
$$

then this coefficient takes the form

$$
g(x, y)=\frac{x y-\xi y^{2}}{x+\xi}
$$

while the condition becomes

$$
x(1-\xi x-y)=0 \Rightarrow y=1-\xi x .
$$

Note that $\mathrm{x}=0$ gives $g \leq 0$ so it is rejected. Using (31), $g$ becomes a function of $x$ only

$$
f(x)=g(x, y(x))=\frac{-\xi\left(1+\xi^{2}\right) x^{2}+\left(1+2 \xi^{2}\right) x-\xi}{x+\xi} .
$$


We find the allowed values of $x$. A natural requirement is $p_{3}\left(T_{f}\right) \geq p_{3}(0) \Rightarrow$ $y(x-\xi y) \geq 0 \Rightarrow(y \geq 0$ and $x-\xi y \geq 0)$ or $(y \leq 0$ and $x-\xi y \leq 0)$. Using (31) we find that the first option implies $x_{1} \leq x \leq x_{2}$ and the second $x_{2} \leq x \leq x_{1}$, where $x_{1}=\xi /\left(1+\xi^{2}\right), x_{2}=1 / \xi$. Since $x_{1}<x_{2}$, only the first option is acceptable, so it must be $x \in\left[x_{1}, x_{2}\right]$. For such $x$, the similar requirement $p_{1}\left(T_{f}\right) \leq p_{1}(0) \Rightarrow x+\xi \geq 0$ is satisfied. So we maximize $f$ in the interval $\left[x_{1}, x_{2}\right]=\left[\xi /\left(1+\xi^{2}\right), 1 / \xi\right]$. Solving the equation $f^{\prime}\left(x_{0}\right)=0$, we find

$$
x_{0}=\sqrt{\xi^{2}+2}-\xi .
$$

Indeed, $x_{0} \in\left[x_{1}, x_{2}\right]$. The corresponding maximum value of $f$ is

$$
f_{\max }=\frac{x_{0}^{4}}{4} .
$$

The maximum achievable value of $p_{3}$ is

$$
p_{3}\left(T_{f}\right)=p_{3}(0)+f_{\max } p_{1}(0) .
$$

Condition (28) implies that in the optimal case it is $\dot{p}_{2}=0$, so it is also $\dot{r}_{2}=0$. If $r_{2}(0)=0$ then $r_{2}(t)=0$ and, as we can see from (15), there is no transfer from $r_{1}$ to $r_{3}$. What we actually examine here is the limiting case $r_{2}(0)=\epsilon \rightarrow 0^{+}$, where $\epsilon$ is an arbitrarily small positive number. We can still use condition (28), i.e. $\dot{r}_{2}=0$. The transfer $r_{1} \rightarrow r_{3}$ takes place through $r_{2}$ which is held to the small constant value $r_{2}=\epsilon$. The maximum achievable value of $r_{3}$, which corresponds to the limit $\epsilon \rightarrow 0^{+}$, is

$$
r_{3}(\infty)=\sqrt{r_{3}^{2}(0)+f_{\max } r_{1}^{2}(0)} .
$$

If the starting state is the point $(1, \epsilon, 0)$, where $\epsilon \rightarrow 0^{+}$, the maximum efficiency is

$$
r_{3}(\infty)=\sqrt{f_{\max }}=\frac{x_{0}^{2}}{2}=\frac{\left(\sqrt{\xi^{2}+2}-\xi\right)^{2}}{2} .
$$

For $\xi=1$ we find that this efficiency is $2-\sqrt{3}$. In Fig. [6 we plot the optimal controls $u_{1}(t), u_{2}(t), u_{3}(t)$, the state variables $r_{1}(t), r_{2}(t), r_{3}(t)$ and the optimal trajectory in r-space. Observe that the starting point is actually $(1, \epsilon, \epsilon)$. It is necessary to give a small positive initial value to $r_{3}$, since the point $(1, \epsilon, 0)$ is still a stationary point of the optimal policy. If the starting point is $(1, \epsilon, \epsilon)$, then by solving the corresponding semidefinite program we find numerically the same efficiency as in (37), in the limit $\epsilon \rightarrow 0^{+}$. 


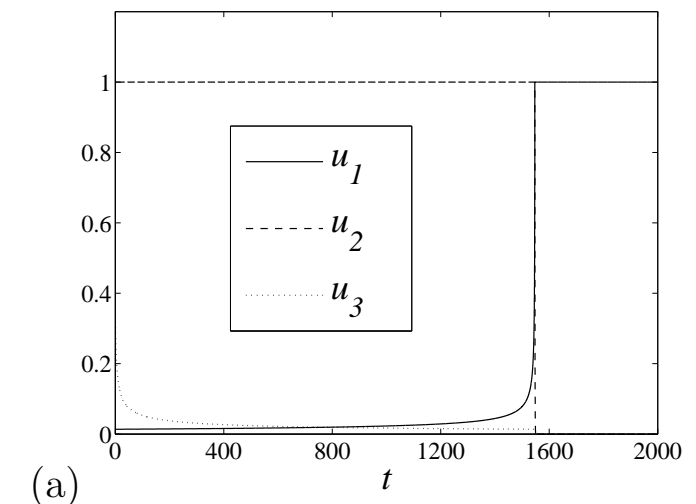

(a)

(b)

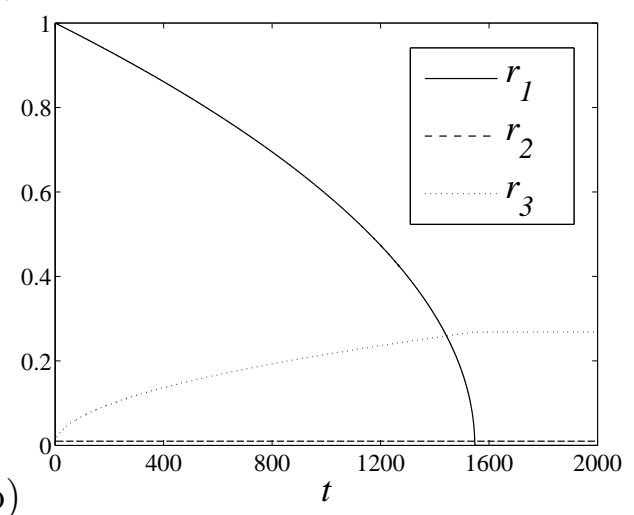

(c)

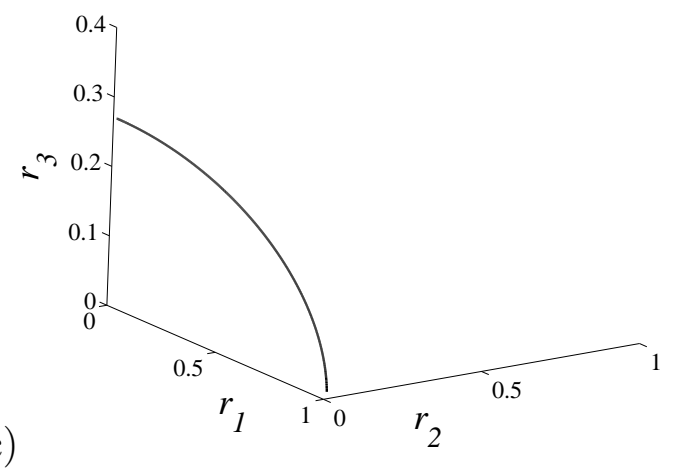

Figure 6: (a) Optimal controls $u_{1}(t), u_{2}(t)$ and $u_{3}(t)$ for system (5), with $A$ the $3 \times 3$ matrix given in the text, when $\xi=1$ and $\left(r_{1}(0), r_{2}(0), r_{3}(0)\right)=$ $(1, \epsilon, \epsilon), 0<\epsilon \ll 1$. Here we take $\epsilon=0.01$ for convenience (b) The corresponding state variables $r_{1}(t), r_{2}(t)$ and $r_{3}(t)$. Note that transfer $r_{1} \rightarrow r_{3}$ takes place through $r_{2}$ which is held to the small constant value $r_{2}=\epsilon$. Thus, this transfer requires more time compared to the preceding examples (c) The optimal trajectory in $r$-space. 


\section{Conclusion}

In this paper we studied a class of bilinear control systems, motivated by optimal control problems arising in the context of dissipative quantum dynamics. It was shown that the optimal solution and the reachable set of these systems can be found by solving a semidefinite program. As a practi-

cal result, solutions to these problems give upper bounds for the ability to coherently control quantum mechanical phenomena in presence of dissipation. In the area of coherent spectroscopy, these results translate into the maximum signal that can be obtained in an experiment. The paper also motivates the use of semidefinite programming to study reachable sets of more general bilinear control systems.

\section{References}

[1] N. Khaneja, T. Reiss, B. Luy, and S. J. Glasser, "Optimal control of spin dynamics in the presence of relaxation", J. Magn. Reson., vol. 162, no. 2, pp. 311-319, June 2003.

[2] A. Barvinok, A Course in Convexity. Providence, RI: American Mathematical Society, 2002.

[3] K. C. Toh, M. J. Todd, and R. H. Tütüncü, "SDPT3 - a Matlab software package for semidefinite programming", Optimization Methods and Software, vol. 11, pp. 545-581, 1999.

[4] D. Stefanatos, N. Khaneja, and S. J. Glasser, "Relaxation optimized transfer of spin order in Ising spin chains", e-print quant-ph/0505116. 